\title{
Upgrading Legacy Equipment to Industry 4.0 through a Cyber-Physical Interface
}

\author{
Hanna Jónasdóttir ${ }^{1}$, Karishma Dhanani ${ }^{1}$, Kenneth McRae ${ }^{1}$ and Jörn Mehnen ${ }^{1(\varpi)}$ \\ ${ }^{1}$ University of Strathclyde, Glasgow G1 1XQ, UK \\ hanna.jonasdottirestrath.ac.uk \\ karishma.dhananiestrath.ac.uk \\ k.mcraedstrath.ac.uk \\ jorn.mehnendstrath.ac.uk
}

\begin{abstract}
With the recent developments of Industry 4.0 technologies, maintenance can be improved significantly by making it "smart", proactive and even self-aware. This paper introduces a new cutting-edge interfacing technology that enables smart active remote maintenance right on the machine in real-time while allowing integration of smart automated decision making and Industrial Internet of Things to upgrade existing legacy equipment through latest Industry 4.0 technology. This interfacing technology enables remote sensing and actuation access to legacy equipment for smart maintenance by entirely non-intrusive means, i.e. the original equipment does not have to be modified. The design was implemented in a real-world manufacturing environment.
\end{abstract}

Keywords: Industry 4.0, Legacy Machine Upgrade, Cyber-Physical Interface.

\section{$1 \quad$ Introduction}

Ever since the first industrial revolution in the $18^{\text {th }}$ century, industry has been constantly evolving and looking for solutions to increase efficiency and reduce cost. With emerging technologies, such as Big Data, Industrial Internet of Things (IIoT) and Augmented Reality (AR), industry can gain more insight into its processes than it has ever done before. One of the areas that are benefitting from this development is maintenance.

Historically, maintenance can be divided into three categories, i.e. unplanned maintenance, planned maintenance and condition-based maintenance (CBM), where CBM is the most recent development [1]. With the aforementioned technologies, predictive and remote maintenance has been made easier, taking CBM one step further by using data analytics to predict failures. As smart technologies are getting more advanced, self-aware and self-maintained machine systems will be the logical next step [2].

Even though Industry 4.0 (I4) technologies have shown to be beneficial to industry [3], implementing I4 is still a challenge. One major challenge is related to data and data analytics $[4,5]$. Security and model integration is also a major issue that needs to be 
considered when designing and implementing I4 technology in a life industrial environment [4-6].

The practical implementation of embedded devices in existing machine environments often implies the need for manipulation of the target machine. This is often not desirable or possible for e.g. warranty reasons or simply because the process is too difficult, expensive or considered not safe. This need has also been highlighted in industry as companies have started implementing remote control programs in their equipment. By having remote access to machinery, machine downtime has been reduced as well as service cost [7].

This paper introduces a new, non-intrusive technology that leverages the availability of I4 technology for machine tools such as smart sensing and actuation while considering safety and security. The target area for demonstrating this new and in principle generically applicable interfacing technology is smart remote maintenance of CNC machine tools as it enables remote access to the machine from anywhere in the world.

\section{$2 \quad$ Literature review}

Even though some companies have already started implementing Industry 4.0, there is still a long way to go [8]. Several approaches have been proposed to implement I4 technologies in industry. The 5C architecture as introduced by Lee et al. [9] is a stepby-step guideline divided into five layers for the implementation of Cyber-Physical Systems (CPS) in a manufacturing environment. Chien et al. [10] suggested a conceptual framework of Industry 3.5 to bridge the gap between Industry 3.0 and Industry 4.0. RAMI 4.0 [11] was developed to aid the integration of I4. The model is a map based on the Life Cycle Value Stream standard IEC 62890 and Hierarchy Levels standard IEC 62264 and IEC 61512 to address the actors needed for the implementation of I4.

As the definition of I4 varies between countries and research groups, different technologies have been suggested to upgrade factories to I4 level. Kang et al. [12] suggested that the key enabling technologies for I4 are: CPS, Internet of Things (IoT), 3D printing, Big Data, Cloud Computing, sensor, smart energy and holograms. Wang et al. [5] stated that IoT, Big Data, Cloud Computing and Artificial Intelligence (AI) are the main enablers of digitalization of industry. Zhou et al. [4] proposed that I4 could be realized through CPS, IoT technologies, Cloud Computing, Big Data and advanced analytics.

Radziwon et al. [13] define a Smart Factory as a manufacturing solution in a dynamic environment that can resolve production problems with flexible and adaptive processes. As maintenance is one of the factors of a factory life cycle [14], it is one of the key areas that need to be addressed when implementing I4. The emerging technologies allow for a shift towards remote maintenance of legacy equipment. Remote maintenance can be acquired in a few ways, i.e. remote maintenance system which accesses the health parameters of the machine and with remotely controlled robotics [15]. As Kang et al. [9] noted, many companies are still using legacy equipment, so the application guidelines and reference models need to take that into consideration. In this paper the term "legacy" is used in the sense of machine equipment that is not yet I4 
ready and typically requires upgrading through Internet connectivity to allow access to advanced I4 technology. Typically, machine tools that are older than about 10 years will fall into that category, but even more modern machine tools do not utilize Industry 4.0 interconnectivity to its full extent.

Traditional human-machine interfaces (HMI) typically have only one feature, that is to visually display the system's outputs to a mechanical input. As industry moves towards CPS, the most important tools for HMI are mobile devices such as tablets [16]. By implementing tablets, the interaction with the machine moves from being performed on a keyboard or a mouse, to touch-screen interactions. Furthermore, mobile devices allow the user to access multiple machinery using only one device. Pacaux-Lemoine et al. [17] proposed a human-centered approach for the design and evaluation of manufacturing systems such as smart manufacturing systems. Harrison et al. [18] developed an engineering toolset for CPS. As a part of that toolset, CPS environment interfaces were developed, enabling remote access to machinery.

There has been significant research into using individual IoT devices, however, to gain the benefits of IoT, it is vital to incorporate the devices within bigger systems. With the Web of Things (WoT) [19], a device can be given a uniquely identifiable "existence" in the World Wide Web of physical things. WoT devices can be addressed through the web to receive or send data but also to sense and actuate other devices or components.

\section{BAUTA}

Remote maintenance is a key area benefitting from the changes of I4 [20]. With the I4 revolution, there have been many advances in creating devices which enable the integration of computing capabilities with physical objects [21]. This paper introduces BAUTA ("Bauta" is the Italian word for a classic Venetian mask) - an IIoT interface and technology which creates a smart platform to upgrade machines that have not been connected to the Internet yet using a minimal-invasive approach, i.e. the target machine does not need to be opened or manipulated in any ways. Although there are interface technologies such as RS232 [22] or advanced software tools such as MTConnect ${ }^{\circledR}$ [23], these interfaces are either slow or require in-depth technical knowledge or physical manipulation of the target machine.

Brecher et al. [24] suggested that in order for legacy machines to become part of the IIoT, they need to communicate with other systems within the factory as well as worldwide. The BAUTA physical interface (Fig. 1) mimics the interaction of a worker with the target machine preserving all existing safety features and standards around an existing machine. The BAUTA physical interface is also able to monitor specific parameters during any operation as well as interact physically with the target machine.

A systematic design process was used for the design of the device. The device has gone through several design consideration and the version presented in this paper is the second version of the device. The main challenges which had to be overcome in the design of the interface were to ensure a secure connection and selecting the appropriate mechanical actuators for the device. To avoid any exposure to physical attacks with the 
wireless connections within the system, passwords have been implemented at every interface. For this paper a legacy HAAS CNC machine with a standard CNC controller was used as generic representative for similar machine tools.

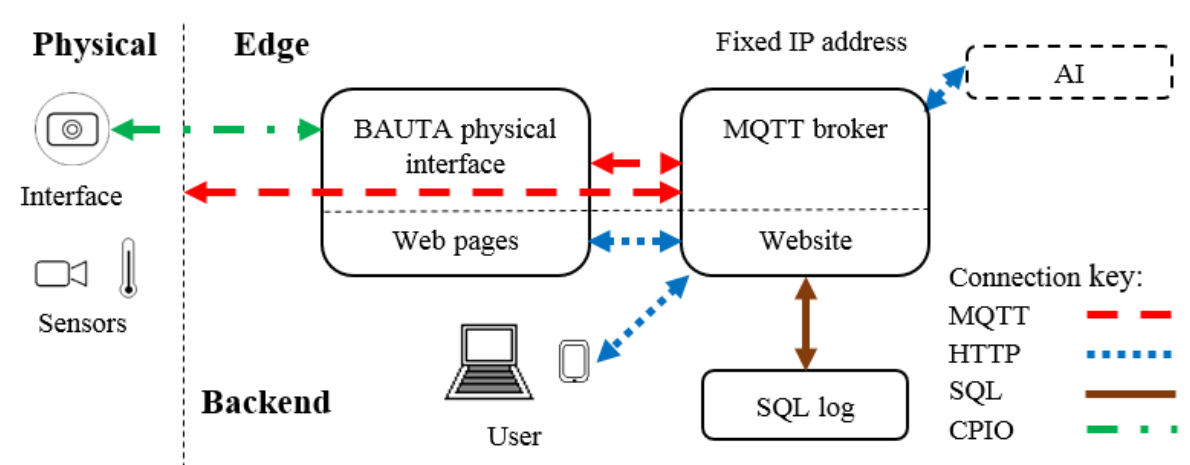

Fig. 1. The BAUTA Cyber-Physical System implementation.

\subsection{Design}

\section{Hardware}

To remotely access legacy machinery, a physical interface is needed to allow the operator to interact with the equipment. To be a practical solution, it needs to be easy to install whilst not compromising the design of the machine or require electrical expertise to fit. To achieve this, the only physical connection used, were bolted mounting points on the controller.

The BAUTA interface mirrors the interaction of a worker with the controller by enabling external actuation of the keys on a $\mathrm{CNC}$ control panel. The design incorporates electro-mechanical actuators which match the key layout of the machine. Arbitrary virtual custom layouts of the keyboard can quickly be created from a template and mapped to the target machine keyboard. The physical BAUTA interface can be designed in parametric solid modelling and printed or machined through additive or subtractive manufacture, respectively. The front of the physical interface features an optional touch screen that allows the operator at the machine to still input commands directly if desired (Fig. 3), which are now mapped through the access software. However, the main task of the physical interface is to map remote commands or command sequences to the physical keyboard while collecting and sending any sensor data back to the remote operator for visual feedback or cloud-based maintenance analytics.

\section{Edge Connection}

For the physical interface to communicate with a web-based interface, the proposed architecture uses an IoT Edge processor to run a middleware software to transfer data. Through WoT technology modular sensors such as vibration, temperature and visual interface can become accessible through the web. The devices are connected through 
$\mathrm{Wi}-\mathrm{Fi}$, as no physical connections are required. For this implementation, an open source Edge processor (Raspberry $\mathrm{Pi} 3 \mathrm{~B}+$ ) was connected through eduroam ${ }^{\circledR}$ allowing remote operation through the academic web.

By following the WoT architecture, the sensors and actuators communicate within the system through MQTT [19], as it allows for a central broker which has a fixed IP address ensuring secure data transfer. The backend uses an HTTP application protocol with WebSockets [19] to transfer the data to and from a website which communicates immediately once the user interacts with the system.

\section{User interface}

For the HMI, a web interface was chosen to allow easy access to any web browsing device as it can be used via any network connection to operate the system with the correct login details. This means that no specialist equipment or software is required. HTML webpages with WebSockets display the information and trends collected from the target machine for the operators to analyze (Fig. 2).

Use of prerecorded macro can be set up for maintenance tasks such as axis datuming and the set-up of feed rates. Setup functions like the loading of G-code from a file can also be processed.

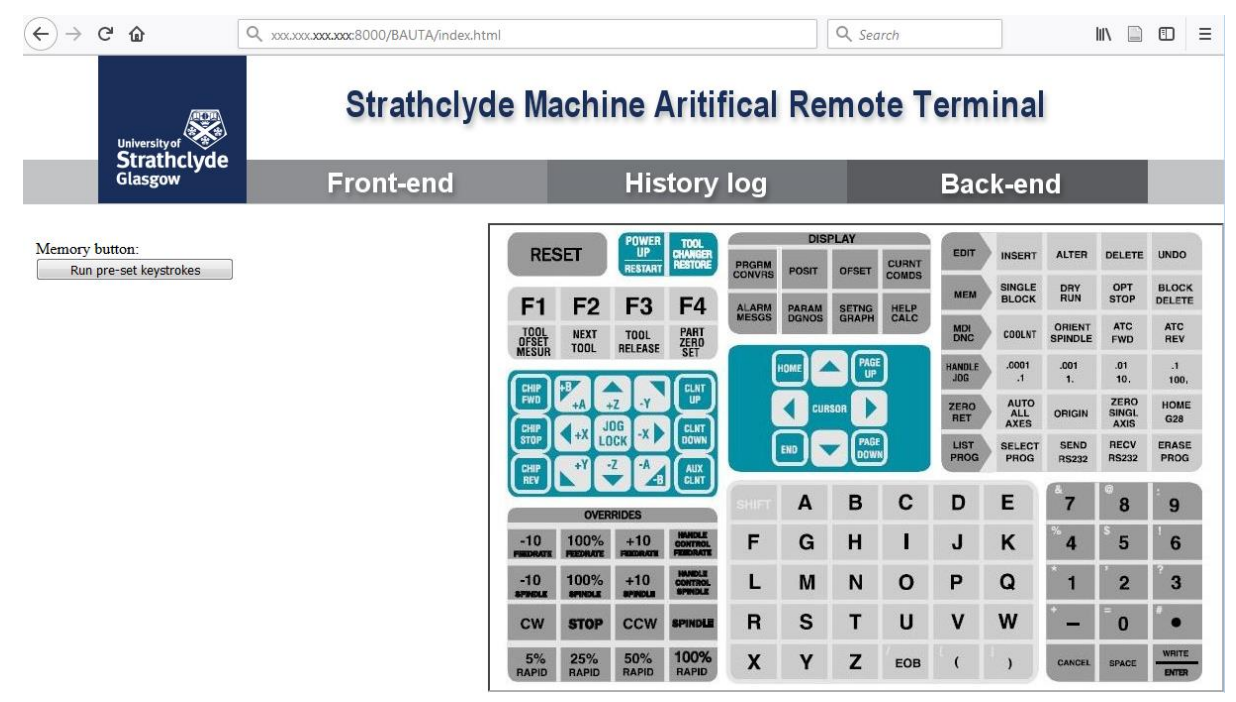

Fig. 2. View of the software displayed on a computer web browser.

\subsection{Implementation}

The physical interface and software was implemented at the Advanced Forming Research Center (AFRC) and University of Strathclyde on a HAAS control panel (Fig. 3). BAUTA successfully connected through eduroam ${ }^{\circledR}$ to the Cloud server and established its connection on the MQTT network automatically. This showed that the device can configure itself over a foreign network, given that it has Internet access. An 
Android ${ }^{\circledR}$ tablet was used to control the CNC machine over the Internet using the Web Interface shown in Fig. 2. Proximity to the machine is not required and that the machine could, in principle, be operated from anywhere in the world. To test the physical device, inputs were actuated from the front panel as shown in Fig. 3. All the trial commands were recorded on the log page along with user comments.

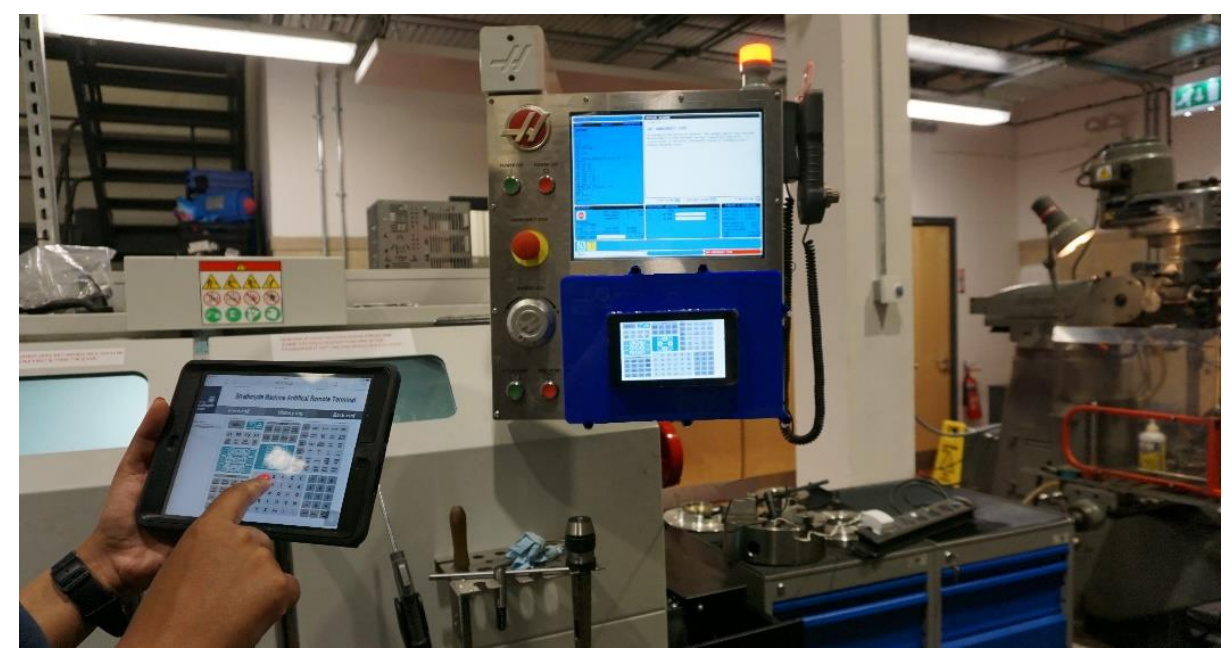

Fig. 3. Remote maintenance interface mounted on a HAAS control panel.

\section{Conclusions}

This paper presents a cutting-edge non-invasive cyber-physical interfacing technology which opens the world of Industry 4.0 through a new cyber-physical overlaying and augmenting approach, which to the authors' best of knowledge is a novel technology. The proposed cyber-physical prototype uses activation keys which can be controlled via the web to allow remote access to the target machine for maintenance activities. The device was successfully implemented on a control panel of a CNC machine, but detailed validation will be carried out in the future.

Future work will include integrating the system with Strathclyde University's new AI enabled smart modular sensors which will also unlock AR/VR capabilities for legacy machine tools. IEC 61499 [25] function blocks could be uploaded to interconnected BAUTA devices to create agile I4-process plans and create G-code in real-time. Furthermore, the design of the BAUTA device can be optimized by following a humancentered design process.

\section{$5 \quad$ Acknowledgements}

This work was supported by the Engineering and Physical Science Research Council (EPSRC) as part of an EPSRC HVMC Fellowship "BAUTA - A Non-invasive remote 
maintenance tool for legacy CNC machine tools". The authors would like to acknowledge the kind support by the Advanced Forming Research Centre (AFRC) at University of Strathclyde.

\section{References}

1. Martin, K.F.: A review by discussion of condition monitoring and fault diagnosis in machine tools. International Journal of Machine Tools and Manufacture 34(4), 527-551 (1994).

2. Lee, J., Kao, H.-A., Yang, S.: Service Innovation and Smart Analytics for Industry 4.0 and Big Data Environment. Procedia CIRP 16, 3-8 (2014).

3. Choi, S., Kim, B.H., Noh, S.D.: A diagnosis and evaluation method for strategic planning and systematic design of a virtual factory in smart manufacturing systems. Int. J. Precis. Eng. Manuf. 16(6), 1107-1115 (2015).

4. Zhou, K., Liu, T., Zhou, L.: Industry 4.0: Towards future industrial opportunities and challenges. In: 2015 12th International Conference on Fuzzy Systems and Knowledge Discovery (FSKD), pp. 2147-2152. IEEE (2015).

5. Wang, S., Wan, J., Li, D., Zhang, C.: Implementing Smart Factory of Industrie 4.0: An Outlook. International Journal of Distributed Sensor Networks 12(1), (2016).

6. Khan, M., Wu, X., Xu, X., Dou, W.: Big data challenges and opportunities in the hype of Industry 4.0. In: 2017 IEEE International Conference on Communications (ICC), pp. 1-6. IEEE (2017).

7. PTC. Reducing Unscheduled Downtime and Customer Efficiency, https://www.ptc.com//media/Files/PDFs/IoT/Axeda-CS-Leica.ashx?la=en\&hash=F070D368C6ABAC9E40D293FB7C698F196D04D295, last accessed 2018/05/26.

8. Qin, J., Liu, Y., Grosvenor, R.: A Categorical Framework of Manufacturing for Industry 4.0 and Beyond. Procedia CIRP 52, 173-178 (2016).

9. Lee, J., Bagheri, B., Kao, H.-A.: A Cyber-Physical Systems architecture for Industry 4.0based manufacturing systems. Manufacturing Letters 3, 18-23 (2015).

10. Chien, C.-F., Hong, T., Guo, H.-Z.: A Conceptual Framework for "Industry 3.5" to Empower Intelligent Manufacturing and Case Studies. Procedia Manufacturing 11, 2009-2017 (2017).

11. Hankel, M., Rexroth B.: The reference architectural model Industrie 4.0 (RAMI 4.0). ZVEI, 2015.

12. Kang, H.S., Lee, J.Y., Choi, S., Kim, H., Park, J.H., Son, J.Y., Kim, B.H., Noh, S.D.: Smart manufacturing: Past research, present findings, and future directions. International Journal of Precision Engineering and Manufacturing - Green Technology 3(1), 111-128 (2016).

13. Radziwon, A., Bilberg, A., Bogers, M., Madsen, E.S.: The Smart Factory: Exploring Adaptive and Flexible Manufacturing Solutions. Procedia Engineering 69, 1184-1190 (2014).

14. Azevedo, A., Almeida, A.: Factory Templates for Digital Factories Framework. Robotics and Computer-Integrated Manufacturing 27, 755-771 (2011).

15. Roy, R., Stark, R., Tracht, K., Takata, S., Mori, M.: Continuous maintenance and the future - Foundations and technological challenges. CIRP Annals 65, 667-688 (2016).

16. Gorecky, D., Schmitt, M., Loskyll, M., Zühlke, D.: Human-machine-interaction in the industry 4.0 era. In: 2014 12th IEEE International Conference on Industrial Informatics (INDIN), pp. 289-294 (2014).

17. Pacaux-Lemoine, M.-P., Trentesaux, D., Zambrano Rey, G., Millot, P.: Designing intelligent manufacturing systems through Human-Machine Cooperation principles: A human-centered approach. Computers \& Industrial Engineering 111, 581-595 (2017). 
18. Harrison, R., Vera, D., Ahmad, B.: Engineering Methods and Tools for Cyber-Physical Automation Systems. Proceedings of the IEEE 104(5), 973-985 (2016).

19. Guinard, D.D., Trifa, V.M.: Building the Web of Things: With Examples in Node.js and Raspberry Pi. Manning (2016).

20. Masoni, R., Ferrise, F., Bordegoni, M., Gattullo, M., Uva, A.E., Fiorentino, M., Carrabba, E., Di Donato, M.: Supporting Remote Maintenance in Industry 4.0 through Augmented Reality. Procedia Manufacturing 11, 1296-1302 (2017).

21. Shariatzadeh, N., Lundholm, T., Lindberg, L., Sivard, G.: Integration of Digital Factory with Smart Factory Based on Internet of Things. Procedia CIRP 50, 512-517 (2016).

22. Electronic Industries Association, Engineering Department: Interface between data terminal equipment and data communication equipment employing serial binary data interchange. Electronic Industries Association, Engineering Dept., Washington (1969).

23. MTConnect ${ }^{\circledR}$ Standard. Part 3.1 - Interfaces, http://www.mtconnect.org/standard-documents-2017/, last accessed 2018/04/05.

24. Jeschke, S., Brecher, C., Meisen, T., Özdemir, D., Eschert, T.: Industrial Internet of Things and Cyber Manufacturing Systems. In: Industrial Internet of Things, pp. 3-19. Springer, Cham (2017).

25. Function Blocks for Industrial-Process Measurement and Control Systems - Part 1: Architecture. IEC 61499, 2005. 